\title{
Desempenho e Solução de Problemas em Equipes Virtuais: Um Estudo Experimental $^{1}$
}

\author{
Sônia Maria Guedes Gondim² \\ Juliana Paranhos Moreno Batista \\ André de Figueiredo Luna \\ Ana Célia Araújo Simões \\ Carolina dos Anjos Almeida Brantes \\ Gisele Debiasi Alberton \\ Universidade Federal da Bahia
}

\begin{abstract}
RESUMO - O estudo analisou as diferenças no desempenho de indivíduos e grupos em uma tarefa de memorização e reprodução de texto em ambiente virtual. Participaram 50 estudantes de nível superior, distribuídos em duas condições experimentais: grupal ( $n=26)$ e individual ( $n=24)$. Utilizaram-se os seguintes instrumentos: crenças e percepção de conflitos sobre o trabalho em equipe, auto e heteroatribuição de estados afetivos e estratégias de solução de problemas. O desempenho grupal na tarefa foi superior ao individual. Participantes na condição individual relataram maior uso de estratégias de solução de problemas que aqueles da condição grupal. Não foram evidenciadas diferenças entre as condições individual e grupal no tocante às crenças e à percepção de conflitos sobre o trabalho em equipe, bem como à atribuição de estados afetivos.
\end{abstract}

Palavras-chave: desempenho de equipe, equipes virtuais, solução de problemas, estudo experimental

\section{Performance and Problem Solving in Virtual Teams: An Experimental Study}

\begin{abstract}
This study examined differences in the performance of individuals and groups on a task of text memorization and reproduction in a virtual environment. Fifty college students were - assigned to one of two experimental conditions: group $(\mathrm{n}=26)$ and individual $(\mathrm{n}=24)$. The instruments applied were: teamwork beliefs and conflict perception, self and heteroattribution of affective states and problem-solving strategies. Group performance was better than individual performance. Participants in the individual condition reported greater use of problem-solving strategies than those in the group condition There were no differences between individuals and groups concerning teamwork beliefs and conflict perception as well as attribution of affective states.
\end{abstract}

Keywords: team performance, virtual teams, problem-solving, experimental study

As organizações têm buscado progressivamente a efetividade de suas equipes para enfrentar a complexidade dos processos de trabalho. A crença predominante é de que as tarefas realizadas em equipe oferecem melhores resultados por utilizarem as diversas habilidades de seus membros. Porém, nem sempre essas diferentes habilidades são bem aproveitadas. As interações entre os membros da equipe, portanto, necessitam ser mais bem estudadas para oferecer insumos para as ações de gestão nas organizações (Potter \& Balthazard, 2002).

Compreender os fatores que contribuem para a eficácia das equipes ao longo do tempo constitui um tema contemporâneo de pesquisa, principalmente no contexto das organizações de trabalho, onde o desempenho da equipe é essencial para o resultado organizacional (Bell \& Marentette, 2011; Hackman, 1987).

A equipe de trabalho pode ser definida como um conjunto dinâmico no qual seus membros possuem características complementares e atuam de modo interdependente, em termos de tarefas e de resultados, para atingir objetivos comuns

1 Apoio: $\mathrm{CNPq}$

2 Endereço para correspondência: Rua Rodrigo Argolo, 293 apto 502, Rio Vermelho, Salvador, Bahia, Brasil, CEP: 41940-220.

Email: sggondim@gmail.com
(Martins \& Puente-Palacios, 2010). A interdependência de tarefas pode ser compreendida como a extensão em que os membros do grupo devem necessariamente interagir para completar o trabalho de maneira satisfatória. A interdependência de resultados é o grau em que se acredita que ganhos e perdas de um indivíduo dependem do resultado dos demais membros de sua equipe (Guzzo \& Shea, 1992; Van der Vegt, Emans, \& Van De Vliert, 2000).

Um estudo multinível realizado por Puente-Palacios e Borges-Andrade (2005) sobre o efeito da interdependência de tarefas e de resultados na satisfação de equipes de trabalho concluiu que crenças individuais na efetividade das equipes atuam conjuntamente com a interdependência de resultados na explicação da satisfação. As pessoas que acreditam nas equipes de trabalho como unidades de desempenho efetivas e que dependem dos colegas para atingir os seus resultados estão mais satisfeitas com a equipe de trabalho do que as que percebem os seus resultados como sendo menos interdependentes dos resultados dos demais membros. Esses achados convergem com os de Saavedra, Earley e Van Dyne (1993), para quem a elevada interdependência de tarefas associada à elevada interdependência de resultados favorece uma maior efetividade da equipe, quando comparada à elevada interdependência de tarefas associada à baixa interdependência de resultados ou vice-versa. Autores como, 
por exemplo, Van Der Vegt et al. (2000), argumentam que no caso de elevada interdependência de resultados, o grupo claramente se beneficia.

É importante salientar que a interdependência de tarefas é uma condição necessária, mas não suficiente da equipe de trabalho, pois é preciso também que haja algum nível de interação entre os membros para a realização da tarefa. Destarte, embora no processo de produção em massa haja tarefas interdependentes, elas nem sempre exigem interação, o que caracteriza um trabalho em grupo, mas não uma equipe de trabalho propriamente dita (Greenberg \& Baron, 1995; Puente-Palacios \& Albuquerque, 2014).

À medida que a complexidade da tarefa aumenta, os membros precisam compartilhar mais suas habilidades e, então, o nível de interdependência na tarefa passa a ser mais elevado, sendo maior ou menor a depender das características individuais. A maior interdependência da tarefa, no entanto, pode, em princípio, contribuir para a emergência de conflitos. De Dreu e Weingart (2003) afirmam que o conflito interfere menos em tarefas mais simples e rotineiras do que em tarefas mais complexas que requerem maior capacidade cognitiva e mais tempo para execução.

A percepção de interdependência nos resultados, no entanto, parece estar associada à interdependência das tarefas. Se uma equipe percebe que o resultado na realização de uma tarefa será compartilhado por todos, tenderá a aumentar as interações e repartir mais adequadamente os recursos entre os membros para realizar a tarefa (Guzzo \& Shea, 1992).

Outras variáveis estão associadas à efetividade da equipe de trabalho: (a) contexto em que está inserida; (b) metodologia de trabalho; (c) equidade na distribuição de tarefas; (d) habilidades complementares dos membros para atingir os objetivos; (e) suporte material e social para o funcionamento das equipes; (f) sistema de recompensas; (g) feedback de desempenho; (h) afetos positivos no grupo; e (i) eficácia do resultado obtido (Puente-Palacios \& Albuquerque, 2014; Martins \& Puente-Palacios, 2010).

Com o desenvolvimento tecnológico e a intensificação da comunicação eletrônica, uma nova modalidade de trabalho em equipe vem crescendo nas organizações. Caracterizada como uma equipe do tipo virtual, distingue-se de uma equipe presencial por duas características: (a) prevalência da comunicação mediada por computadores (CMC) (Anawati \& Craig, 2006; Griffith \& Neale, 2001), e (b) dispersão geográfica parcial ou total de seus membros (Ahuja \& Carley, 1999; Griffith \& Meader, 2004).

As equipes virtuais rompem as fronteiras geográficas e fazem crescer as redes organizacionais, contribuindo para uma nova configuração dos processos de trabalho. Sua popularidade cresce, pela flexibilidade e poder de fazer ampliar as atuações das organizações no mundo (Bell \& Kozlowski, 2002; Kayworth \& Leidner, 2002; Rangolam \& Ballard, 2007). Em um ambiente virtual, a interação (social ou voltada para a tarefa) tem um papel central no desempenho da equipe. O desempenho na realização da tarefa requer troca de informação eficiente e comunicação clara e sistemática, permitindo que sistemas e pessoas interajam para garantir a finalização da tarefa e o alcance dos objetivos. As dinâmicas de trabalho, no entanto, nem sempre permitem que todos estejam atualizados sobre as informações necessárias à execução do trabalho individual, alinhado aos objetivos do grupo, da empresa ou de uma rede organizacional. A comunicação mediada por computador supera em parte as barreiras no fluxo de informações, em organizações de grandes dimensões ou cujas tarefas estejam dispersas geograficamente.

Embora as equipes de trabalho tenham ganhado popularidade nas organizações, particularmente as equipes virtuais que permitem que a tarefa seja realizada por formas mediatas de comunicação, sua efetividade depende de inúmeras variáveis, dentre as quais se destacam as estratégias de solução de problemas utilizadas na realização de tarefas, as crenças na eficácia do trabalho em equipe, a percepção de conflitos entre os membros e os estados afetivos de seus membros. As seções subsequentes irão abordar cada um destes tópicos.

\section{Estratégias para solução de problemas}

Os obstáculos no alcance dos objetivos da equipe motivam seus membros a se engajarem na busca de solução de problemas. Isso é fundamental para a tomada de decisões na realização de tarefas (Sauaia \& Zerrener, 2009).

Na perspectiva de Sternberg (2000) são sete as etapas do ciclo de resolução de problemas. A primeira é a identificação do problema, cujo foco é demarcar os obstáculos, objetivos e recursos disponíveis. A segunda etapa é a definição e representação do problema, sendo preciso defini-lo e representá-lo de maneira que se possa entendê-lo e resolvêlo, evitando soluções inadequadas. Os problemas podem ser mal ou bem estruturados em virtude da quantidade de informações disponíveis para sua solução. Em outras palavras, quanto mais se encontram estabelecidas as regras e condições do problema, mais bem estruturado ele é, deixando as alternativas de resolução mais claras e evidentes. Em problemas mal estruturados, torna-se necessário proceder a uma busca ativa por informações para solucioná-los.

A terceira etapa refere-se à escolha da estratégia de resolução de problemas: análise, pensamento convergente e pensamento divergente. A análise consiste em decompor um problema em elementos manejáveis e é acompanhada de uma síntese que os reintegra de modo lógico. O pensamento divergente e o convergente são definidos como duas estratégias criativas na resolução de problemas. No primeiro, procura-se identificar todos os pontos relacionados à situação, e no segundo, procura-se avaliar as alternativas elencadas e selecionar as mais viáveis. A quarta etapa é a organização estratégica da informação para a execução da alternativa escolhida. O quinto estágio é o da alocação de recursos cognitivos para resolver a tarefa. Quanto mais o indivíduo despende recursos mentais para o planejamento, mais tempo e energia tende a economizar na sua execução, diminuindo a probabilidade de frustração. A sexta etapa é a do monitoramento do processo, em que se avalia quão próximo se está de alcançar o objetivo da tarefa. A sétima e última etapa é a da avaliação da solução encontrada.

Embora nem sempre os processos de tomada de decisão para realização de tarefas em equipes sigam essas etapas de solução de problemas, parte-se da premissa de que a 
quantidade de estratégias usadas permite inferir o nível de investimento cognitivo mobilizado pelos membros da equipe para solucionar o problema, afetando o desempenho da equipe.

\section{Crenças}

Uma segunda variável que está relacionada ao desempenho de equipes são as crenças sobre sua eficácia. As crenças expressam a relação subjetiva de credulidade que a pessoa estabelece com o objeto da crença e resultam de suas experiências prévias, positivas ou negativas, e do conhecimento que se adquire na relação com o mundo. $\mathrm{O}$ bom desempenho de uma equipe de trabalho depende de crenças congruentes, tanto entre os membros quanto entre eles e a organização (Wageman, 1995).

A crença na eficácia do trabalho em equipe auxilia os membros a pensarem de modo estratégico, a tomarem decisões e a enfrentarem os obstáculos (Bandura, 2000). As crenças também são preditores do desempenho da equipe (Lent, Schmidt, \& Schmidt, 2006).

As crenças e os conflitos estão bastante relacionados, visto que conflitos entre os membros da equipe podem resultar em crenças negativas acerca do desempenho da equipe em comparação ao desempenho individual (Martins \& Puente-Palacios, 2010). Por outro lado, equipes com crenças mais positivas sobre o trabalho em equipe tendem a lidar melhor com o conflito e a trabalhar de forma mais produtiva (Alper, Law, \& Tjosvold, 2000).

\section{Conflitos}

Uma terceira variável importante para entender o funcionamento de uma equipe é o conflito. Para De Dreu e Weingart (2003), o conflito de equipe é desencadeado pela percepção das diferenças entre seus membros, podendo ser de dois tipos: de relacionamento e de tarefa. O primeiro tipo se refere às desavenças interpessoais, e o segundo, à discordância dos procedimentos adotados na realização de tarefas e atividades (Gamero, González-Romá, \& Peiró, 2008; Martins, \& Puente-Palacios, 2010).

Embora o conflito seja visto como uma variável que interfere de forma negativa nas equipes de trabalho, os resultados empíricos têm sido inconsistentes. Um baixo nível de conflito de tarefa pode ser benéfico para a equipe, porque sinaliza haver uma interação positiva entre os membros. Por outro lado, as discordâncias quanto à tarefa podem ampliar a análise do problema, levando a melhores soluções (Gamero et al. 2008; Martins \& Puente-Palacios, 2010). O conflito de tarefa pode também contribuir para o conflito de relacionamento, prejudicando a efetividade da equipe de trabalho (De Dreu \& Weingart, 2003; Gamero et al., 2008; Martins \& Puente-Palacios, 2010).

Conforme referido anteriormente, o conflito pode ser tanto desencadeado por discordâncias acerca da realização de tarefas quanto por tensões nas relações interpessoais. Em ambas as situações encontram-se implicados estados afetivos dos membros da equipe. Disposições emocionais iniciais e afetos desencadeados pelas circunstâncias emergentes nas interações de trabalho também contribuem para a efetividade das equipes.

\section{Estados afetivos}

Afetos podem ser concebidos como uma categoria ampla que abarca emoções, sentimentos, humores e temperamentos. As diferenças entre os diversos afetos estariam marcadas por sua duração, pela prontidão de resposta e pela experiência subjetiva. As emoções ou estados afetivos são manifestações de curta duração e os sentimentos estão associados à experiência subjetiva. Os humores são estados afetivos desencadeados por eventos momentâneos, que se transferem a outros objetos, fazendo-os perdurar por mais tempo. O temperamento está mais associado a traços pessoais duradouros que se refere ao modo afetivo de ser da pessoa (Gondim \& Álvaro-Estramiana, 2010; Gray \& Watson, 2001). Os estados afetivos são processos influenciados pelo contexto social, variando de acordo com as demandas ocupacionais, organizacionais e pessoais, e pela socialização e reações dos demais indivíduos.

Ao analisar estudos que relacionam emoções e situações de aprendizagem online, Wosnitza e Volet (2005) concluíram que atividades de treinamento percebidas como desconhecidas, desafiadoras e relevantes para o aprendiz ativam emoções tanto de intensidade positiva quanto negativa. Dito de outro modo, novos desafios podem fazer com que altos níveis de excitação sejam acompanhados de altos níveis de ansiedade. No estudo de Barsade (2001) sobre a repercussão de afetos no trabalho em equipe, o contágio emocional positivo se correlacionou positivamente ao comportamento cooperativo no grupo e ao desempenho nas tarefas, e negativamente com o nível de conflito no grupo. Bartel e Saavedra (2000) apontaram que humores agradáveis aumentam a qualidade e a eficiência do desempenho do grupo. Em contrapartida, humores desagradáveis motivam o grupo a alcançar seus objetivos e a fornecer a quantidade de produtos ou serviços esperados.

Para alguns teóricos, como Csikszentmihalyi e LeFevre (1989), existe uma relação entre os estados afetivos vivenciados em tarefas desafiadoras e a percepção de dificuldades e de competências pessoais necessárias para enfrentá-los. Em caso de equilíbrio entre os dois componentes, supõe-se haver motivação intrínseca necessária para alcançar um nível de excitação que gere estados afetivos positivos para assegurar o sucesso na tarefa.

Ao levar em conta as inúmeras variáveis que contribuem para o êxito no desempenho das equipes, o estudo experimental descrito neste artigo comparou indivíduos e grupos, visando ajudar a elucidar as relações entre, de um lado, as crenças no trabalho em equipe, percepção de conflitos intragrupal e estados afetivos individuais e, de outro, o desempenho na realização de tarefa. A adoção crescente das equipes virtuais pelas organizações atuais suscitou o interesse em explorar as relações entre os construtos supracitados em um desenho experimental em que estudantes universitários na condição individual ou em grupo realizaram uma tarefa de memorização e reprodução de um texto de modo 
colaborativo, mediante o uso de computadores. Testou-se a influência da condição de realização da tarefa (individual ou em grupo) sobre o desempenho e o uso de estratégias de solução de problema. Avaliou-se também se, após a realização da tarefa, as crenças na eficácia do trabalho em equipe, na percepção de conflitos e nos estados afetivos iniciais dos estudantes apresentavam mudanças.

\section{Método}

Trata-se de um estudo experimental em que a variável independente foi a forma de realização da tarefa (individual ou em equipe). As variáveis dependentes foram os estados afetivos, o desempenho na tarefa, as crenças e, por último, a percepção de conflitos sobre o trabalho em equipe.

\section{Participantes}

Participaram da pesquisa 50 estudantes universitários, 30 mulheres e 20 homens, com idade média de 24,13 anos $(\mathrm{DP}=3,9)$. A maioria possuía nível superior incompleto $(88 \%)$ e estavam vinculados principalmente às áreas de ciências sociais e de humanas (92\%). Do total, 24 foram alocados na condição individual e 26, na grupal. A amostra foi por critério de conveniência. Os participantes expressaram sua concordância em participar da pesquisa por meio do Terno de Consentimento Livre e Esclarecido (TCLE), em conformidade com as normas de ética vigentes no país

\section{Ambiente e equipamento}

O experimento foi conduzido no Laboratório de Cognição Social (sala de $6 \times 4 \mathrm{~m}$ ) de uma instituição pública federal de Ensino Superior. A sala continha uma mesa, um notebook conectado a uma caixa de som, duas cadeiras para os dois experimentadores e quatro estações de trabalho dotadas, cada uma, de um computador com uma câmera de vídeo acoplada e uma cadeira. As estações de trabalho eram separadas por uma divisória em fórmica. Na parede em frente a cada estação de trabalho havia um cartaz que informava o código de identificação do grupo de cada sessão a ser digitado pelo participante ao iniciar o experimento. Foi utilizada também uma sala anexa ao laboratório $(4 \times 3 \mathrm{~m})$ para a recepção dos participantes que continha apenas cadeiras dispostas em forma de "U".

\section{Instrumentos}

Foi usado o software EFS (Enterprise Feedback Suite), produzido pela Globalpark $\mathrm{GmbH}$, para condução de pesquisas online. Os instrumentos utilizados, apresentados em versão eletrônica para os participantes, são descritos a seguir.

Escala de Crenças sobre o Trabalho em Equipe (Van der Vegt, Emans, \& Vliert, 1999): A medida foi adaptada ao contexto brasileiro por Puente-Palacios (2002) e usada na pesquisa sobre o psicólogo no Brasil (Bastos \& Gondim, 2010). Possui quatro itens (e.g., "Considero um bom investimento o tempo gasto no trabalho em equipe."), respondidos em escala crescente de concordância de cinco pontos. A estrutura é unifatorial e a confiabilidade é satisfatória $(\alpha=0,75)$. No presente estudo, a escala de resposta foi de sete pontos, variando de 1 (discordo totalmente) a 7 (concordo totalmente); o índice de confiabilidade (Alpha de Cronbach) antes da realização da tarefa foi de 0,67 e depois, de 0,69 .

Escala de Percepção de Conflitos no Trabalho em Equipe (Jehn, 1994): O instrumento foi traduzido e adaptado ao contexto brasileiro por Martins, Guimarães e Oliveira (2006). Contém oito perguntas, tais como: “Quanto conflito há entre os membros de sua equipe ou equipe de trabalho?" e "Quanta divergência de opinião existe em sua equipe ou equipe de trabalho?". A escala possui dois fatores: conflitos de relacionamento e de tarefa. Em pesquisa realizada com 253 psicólogos brasileiros, Martins e Puente-Palacios (2010) obtiveram uma estrutura unifatorial. Esse fator foi denominado "Conflito Intragrupal" e obteve índice satisfatório de confiabilidade $(\alpha=0,86)$. A presente pesquisa também utilizou o instrumento com os oito itens que foram respondidos mediante escala de cinco pontos que variava de 0 (Nenhum) a 4 (Muitíssimo). A confiabilidade da escala (Alpha de Cronbach) foi 0,63 (antes da realização da tarefa) e 0,81 (depois da realização da tarefa).

Medidas de Atribuição Pessoal de Estados Afetivos (adaptada de Gondim \& Mutti, 2011) e de Emoções Básicas (baseada em Ekman, 1992). A atribuição de estados afetivos foi medida a partir de uma lista de 22 afetos, sendo 11 deles positivos (e.g., surpresa agradável e bem-estar) e 11 negativos (e.g., ansiedade, tristeza e raiva). Antes da realização da tarefa, os participantes eram convidados a se autoatribuírem afetos vivenciados nas últimas semanas, medidos por uma escala de sete pontos, na qual 1 representava ausência do afeto e 7 indicava que o afeto estava muito presente. A mesma medida foi aplicada após a realização da tarefa.

A atribuição de emoções básicas foi medida somente após a realização da tarefa. Eram apresentadas cinco emoções básicas (alegria, tristeza, raiva, medo e nojo), sendo solicitado aos participantes que observassem a sua imagem e a dos demais participantes da sessão na tela do computador (imagens captadas por webcams) e fizessem atribuições da intensidade das emoções para cada um deles. Utilizou-se uma escala em que a atribuição a cada emoção básica variava de 1 (nada) a 7 (muito).

Estratégias de Solução de Problemas (instrumento baseado em Sternberg, 2000). Esse instrumento foi elaborado pela equipe de pesquisa, com base no Ciclo de Resolução de Problema de Sternberg (2000) e considerando as etapas propostas pelo autor: identificação e representação do problema, definição das estratégias utilizadas para a solução do problema, organização das informações, alocação dos recursos e avaliação da solução encontrada. O instrumento possui 13 questões gerais sobre soluções de problemas (e.g., "A atenção foi focada nas informações relevantes desprezando-se as irrelevantes"), três sobre as estratégias 
utilizadas (e.g., "Decomposição do problema em partes menores") e quatro sobre o quanto cada item dificultava ou facilitava o trabalho em equipe (e.g., "Forma de definição do problema"). Para essas últimas quatro questões, a escala foi de sete pontos (1 'dificultou' e 7 'facilitou'). Para as demais questões, utilizou-se uma escala de quatro pontos de 1 (nada) a 4 (muito).

Para medir o desempenho na tarefa, duas outras variáveis foram criadas com base nos textos produzidos pelos participantes do experimento. Uma delas foi a eficácia de memorização, a qual avaliava se o número de palavras do texto produzido era equivalente aos do texto original. Para definir parâmetros de medida, todas as palavras do texto foram listadas e 67 palavras-chave foram selecionadas (e.g., "acidente", "Alicante", "Logan" e "vôo"). Algumas se repetiam, totalizando 81 palavras que foram utilizadas como base de cálculo para elaboração do critério. O número de acertos foi classificado em quatro intervalos de desempenho. Os intervalos eram até $24 \%$ ( $\leq 19$ palavras corretas), de 25 a $49 \%$ (20 a 39), de 50 a $74 \%$ (40 a 60) e entre 75 a $100 \%$ (61 a 81), dando origem, respectivamente, à codificação: 1 (desempenho ruim), 2 (desempenho regular), 3 (desempenho bom) e 4 (desempenho excelente). Adotou-se como critério de acerto o radical comum da palavra, por exemplo, se a palavra original era "treino", aceitava-se "treinamento".

A outra variável de desempenho foi eficácia de apreensão de conteúdo, que avaliou o conteúdo do texto apresentado pelos estudantes (desempenho na tarefa) com base nas informações centrais do texto original. Para servir de gabarito de correção, a equipe de pesquisa selecionou as ideias centrais do texto e definiu quatro critérios com pontuações variadas em função de sua relevância e quantidade de vezes que apareciam no texto original.

Para fins de avaliação do êxito no desempenho, a ordem na qual as informações apareciam no texto reproduzido não foi relevante, sendo considerado apenas se o conteúdo estava presente. A pontuação total possível de ser obtida, incluindo todas as informações, era de 12,6 pontos. A avaliação do participante era calculada com base no número de informações descritas nos textos, multiplicado pela pontuação dada a cada critério. O resultado final do indivíduo ou do grupo (a depender da condição) era classificado em quatro categorias: 1 ( 0 a 3,0 pontos, desempenho ruim), 2 (3,1 a 6,0 pontos, desempenho regular), 3 (6,1 a 9,0 pontos, desempenho bom) e 4 (9,1 a 12,6 pontos, desempenho excelente).

\section{Procedimento}

Foram realizadas 13 sessões coletivas (com três ou quatro participantes realizando a tarefa simultaneamente): sete sessões na condição grupal e seis sessões na condição individual. A sessão compreendia a realização da tarefa proposta (memorização e reprodução de um texto) e durava 40 minutos. Cada estudante participava de apenas uma sessão. Ao chegarem ao local de realização da pesquisa, os participantes de cada sessão (três ou quatro) eram direcionados para uma sala anexa ao laboratório. Em seguida, eram convidados a preencher uma ficha em que deveriam registrar se conheciam os demais participantes presentes naquela sala. Só ocorreria alocação na condição grupal se nenhum dos participantes da sessão se conhecesse previamente. Esse controle era para evitar que relacionamentos anteriores pudessem influenciar os resultados de participantes alocados em uma condição em que havia colaboração mediada por computadores. Para os participantes da condição individual o conhecimento prévio não influenciava, visto que, embora compartilhassem a mesma sessão, não interagiam para a realização da tarefa.

Finalizada essa etapa de atendimento ao critério de alocação, os participantes eram conduzidos ao laboratório e orientados a escolher um dos quatro computadores disponíveis. Os computadores estavam equipados com câmeras instaladas e em funcionamento, permitindo que os participantes se vissem via GVO Conference (uma ferramenta de vídeo conferência). Durante a explanação inicial, as câmeras permaneciam funcionando. Essa explanação consistia em uma gravação em áudio, feita previamente por um dos pesquisadores, que informava os objetivos e as instruções de cada etapa do experimento a todos os participantes da sessão simultaneamente. Como não havia ruído no laboratório, fez-se uso apenas de uma caixa de som, não tendo sido necessário o uso de fones de ouvido individuais.

A sessão experimental compreendia cinco tempos (ver Figura 1). No tempo 1 foi apresentado o Termo de Consentimento Livre e Esclarecido na tela do computador de cada participante. Somente após os participantes assinalarem o campo de concordância no referido termo, é que era iniciado o experimento. Em seguida, os participantes responderam a um survey eletrônico com os instrumentos de autoatribuição de estados afetivos (lista de 22 itens), percepção de conflitos e crenças em relação ao trabalho em equipe. Logo após completarem o preenchimento do survey, os participantes foram direcionados novamente para a ferramenta de vídeo conferência, por meio da qual foi apresentado, por 5 minutos, um texto de 150 palavras com informações de datas, nomes e números.

No tempo 2 foi realizada a tarefa, que consistia em reproduzir o texto, sem nenhum tipo de consulta. O Google Docs (documento compartilhado) foi usado no experimento. Para os participantes da condição individual, a tarefa foi realizada sem compartilhamento, e para a condição grupal, o texto era compartilhado, permitindo coedição simultânea entre os componentes do grupo e comunicação entre os membros do grupo via mensagem de texto. Após o término do tempo de realização de tarefa, o texto original era reapresentado no GVO Conference para que os participantes pudessem avaliar a exatidão de seu próprio desempenho. No tempo 3, os estudantes eram convidados a retornar ao survey para responder, além dos três instrumentos anteriores (crenças, conflitos e estados afetivos), a um conjunto de itens sobre o ciclo de solução de problemas.

No tempo 4, a ferramenta de conferência online foi utilizada novamente para exibir as imagens em tempo real dos participantes por cerca de 2 minutos. No tempo 5 foi solicitado que os participantes (condição individual e grupal) atribuíssem emoções básicas (alegria, medo, tristeza, nojo, 


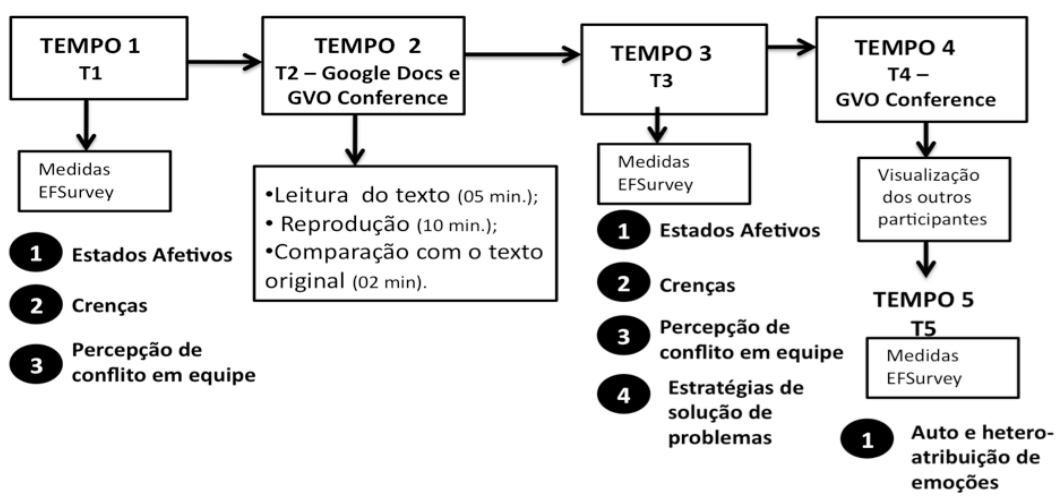

Figura 1. Modelo esquemático da realização do experimento nos cinco tempos. Os participantes em ambas as condições (individual e grupo) responderam ao questionário eletrônico contendo as medidas descritas (tempos 1, 3 e 5), realizaram a tarefa (tempo 2) e visualizaram os outros participantes (tempo 4)..

raiva) a si próprios e aos demais participantes de sua sessão com base nas imagens exibidas.

\section{Análise de dados}

Os dados coletados pelos instrumentos foram analisados por meio de um programa estatístico para ciências sociais. Estatísticas descritivas foram utilizadas para caracterização da amostra e análise da distribuição das respostas nas variáveis mensuradas. Para analisar as relações entre as variáveis, foram realizados testes $t$, análise da variância e correlações de Pearson. Análises de confiabilidade foram realizadas para testar a validade das escalas para essa pequena amostra.

\section{Resultados e Discussão}

\section{Estados afetivos (medida de atribuição pessoal de estados afetivos de 22 itens)}

As análises das medidas no tempo 1 (antes da tarefa) indicaram que, no total da amostra, as médias dos estados afetivos positivos (11 afetos) variaram de 3,81 (orgulho) a 5,08 (alívio), e o desvio padrão oscilou de 1,21 a 1,86. As médias das emoções negativas (11 afetos) variaram de 2,46 (surpresa desagradável) a 5,67 (ansiedade) e o desvio padrão de 1,46 a 1,86 . Foi realizado teste t para comparar os estados afetivos entre as condições experimentais, cujo resultado não apontou diferenças significativas para a percepção dos afetos positivos. Houve diferenças para os afetos negativos $[\mathrm{t}(48)=0,392 ; \mathrm{p}<0,05]$, em que a condição de grupo apresentou maior média $(\mathrm{M}=4,58 ; \mathrm{DP}=1,98)$ que a individual $(\mathrm{M}=3,50 ; \mathrm{DP}=1,76)$.

A comparação, por meio do teste $t$, dos estados afetivos positivos, após a realização da tarefa, entre as condições experimentais, revelaram diferenças significativas para o prazer $[\mathrm{t}(48)=2,11 ; \mathrm{p}<0,05]$, sendo a média maior na condição em grupo $(\mathrm{M}=4,85 ; \mathrm{DP}=1,34)$ que na individual
$(\mathrm{M}=3,96 ; \mathrm{DP}=1,34)$. Não houve diferenças significativas em relação aos afetos negativos.

Foram encontradas correlações somente entre estados afetivos positivos e a eficácia de memorização e de apreensão de conteúdo. No caso da memorização, as correlações foram com os estados afetivos prazer $(r=0,34 ; p<0,05)$ e entusiasmo $(\mathrm{r}=0,31, \mathrm{p}<0,05)$. Em relação à eficácia de conteúdo, a correlação encontrada foi somente com prazer $(\mathrm{r}=0,39 ; \mathrm{p}<0,05)$.

Os resultados estão em sintonia com alguns estudos que afirmam que os afetos positivos favorecem a colaboração no trabalho em equipe (e.g., Bartel \& Saavedra, 2000), principalmente por meio do contágio emocional (e.g., Barsade, 2001). As correlações encontradas também permitem inferir que a tarefa na condição de equipe foi capaz de ativar estados afetivos de prazer e entusiasmo que podem ter contribuído para a qualidade da realização da tarefa. (e,g., Csikszentmihalyi \& LeFevre, 1989).

\section{Estratégias de solução de problemas}

A análise da condição experimental em relação às estratégias de solução de problemas e ao desempenho na tarefa, por meio do teste $t$, revelou diferenças significativas entre as condições para o desempenho (eficácia de memorização e de apreensão de conteúdo) e para alguns itens do processo de solução de problemas. Para os itens de solução de problemas que apresentaram diferenças significativas (Tabela 1), as médias foram maiores na condição individual. Nessa condição, os participantes relataram ter feito mais uso das seguintes estratégias para realizar a tarefa: organizar as informações, decompor o problema em partes menores, equilibrar o planejamento e a execução da tarefa, utilizar analogias e partir de observações específicas. Em contrapartida, as médias de eficácia de memorização e apreensão de conteúdo foram maiores na condição grupal. Ou seja, nessa condição, o desempenho dos participantes foi mais efetivo, mesmo em circunstâncias em que o tempo para realizar a tarefa era limitado e não havia familiaridade entre os membros. Esses resultados estão em sintonia com 
outros (e.g., Laughlin \& Ellis, 1986) que mostram que o aproveitamento das habilidades dos membros dos grupos contribui para o desempenho superior da equipe em relação ao desempenho individual.

No entanto, resultado de estudo recente não corrobora esse achado, colocando em dúvida a crença de que a equipe apresenta sempre um desempenho superior. Ao estudarem a solução de problemas de clientes comparando o desempenho individual e de uma equipe de estagiários de uma empresa multinacional de tecnologia de informação, Alves e Gondim (2014) concluíram que o desempenho de um estagiário foi melhor que o desempenho da equipe, que teve a oportunidade de interagir virtualmente para buscar a solução do problema do cliente. Esse resultado acrescenta também outro elemento para a discussão, visto que os estagiários desse último estudo se conheciam.

Em sendo assim, embora estudos empíricos façam menção à importância da familiaridade entre os membros da equipe para o êxito na realização da tarefa (Harrison, Mohammed, McGrath, Florey, \& Vanderstoep, 2003), isso não pode ser generalizado. No presente estudo, a ausência de familiaridade, critério usado para alocação do participante na condição de grupo, não pareceu afetar o desempenho dos grupos.

Em resumo, a Tabela 1 demonstra que as medidas de eficácia de memorização e de apreensão de conteúdo (indicadores de desempenho na tarefa) foram maiores na condição grupal que na individual. $\mathrm{O}$ resultado sugere que embora as pessoas na condição individual tenham crenças de que adotaram boas estratégias para solucionar o problema, na prática o desempenho é aquém do esperado. Na condição grupal, por sua vez, apesar de as pessoas relatarem menor uso de estratégias de soluções de problemas, o que supostamente poderia reduzir as chances de êxito na realização na tarefa, o desempenho foi superior ao da condição individual.

Para subsidiar a avaliação da eficácia de memorização dos participantes, foram criadas três nuvens de palavras: uma do texto original (ver Figura 2a), uma dos textos produzidos em grupo (ver Figura 2b) e outra dos produzidos individualmente (ver Figura 2c). O aplicativo apresenta um efeito visual com base na frequência das palavras. Para criar as nuvens, todos os textos, por condição, foram unidos em um único arquivo. Para que houvesse proporcionalidade entre as nuvens, na condição de grupo os textos foram replicados pelo número de participantes nessa condição, embora se reconheça que isso pode gerar distorção.

$\mathrm{Na}$ Figura 2(a) destacam-se as palavras "acrobacia", "vôo", e "anos" como as três mais frequentes no texto original. Nas demais figuras, essas palavras também estão em destaque, mas na Figura 2(b) é possível observar também os nomes próprios. Isso sugere maior capacidade de memorização por parte dos grupos para a produção do texto, o que indica que a eficácia de memorização é maior em grupo. Esse resultado, assim como os anteriores, indica que o desempenho de grupo foi melhor que o desempenho individual. Sugere, também, que isso pode ter ocorrido em função da coedição simultânea dos membros da equipe, o que teria permitido que cada membro ativasse a sua memória a partir da contribuição dos demais, assegurando, no final, maior proximidade com o texto original. Os participantes alocados na condição individual, ao contrário, só puderam contar com a sua própria memória. Finalmente, esse achado

Tabela 1. Média, desvio-padrão e valores de t das estratégias de soluções de problemas e desempenho na tarefa

\begin{tabular}{|c|c|c|c|c|c|c|}
\hline & \multicolumn{2}{|c|}{$\begin{array}{l}\text { Indivíduo } \\
(\mathrm{N}=24)\end{array}$} & \multicolumn{2}{|c|}{$\begin{array}{l}\text { Grupo } \\
(\mathrm{N}=26)\end{array}$} & \multirow[t]{2}{*}{$t$} & \multirow{2}{*}{$p$} \\
\hline & $M$ & $D P$ & $M$ & $D P$ & & \\
\hline \multicolumn{7}{|l|}{ Estratégia de soluções de problemas } \\
\hline As informações foram organizadas para facilitar a execução da estratégia. & 2,92 & 0,50 & 2,19 & 0,75 & 3,977 & .001 \\
\hline Foi definido claramente do que se tratava a tarefa. & 3,21 & 0,93 & 2,08 & 0,93 & 4,283 & .001 \\
\hline Decomposição do problema em partes menores. & 3,33 & 0,70 & 2,50 & 1,03 & 3,316 & .002 \\
\hline $\begin{array}{l}\text { A atenção foi focada nas informações relevantes e desprezaram-se as } \\
\text { irrelevantes. }\end{array}$ & 3,29 & 0,62 & 2,88 & 0,65 & 2,250 & .029 \\
\hline Houve equilíbrio entre o planejamento e a execução da tarefa. & 2,46 & 0,83 & 1,81 & 0,69 & 3,010 & .004 \\
\hline $\begin{array}{l}\text { Eu acredito que teria mais êxito na tarefa se houvesse interação virtual } \\
\text { com outras pessoas. }\end{array}$ & 3,46 & 0,78 & 2,35 & 0,80 & 4,983 & .001 \\
\hline $\begin{array}{l}\text { Foram utilizadas analogias com problemas anteriores que contribuíram } \\
\text { para a realização da tarefa. }\end{array}$ & 2,17 & 0,96 & 1,38 & 0,57 & 3,525 & .001 \\
\hline $\begin{array}{l}\text { Partiu-se de observações específicas e obteve-se uma conclusão provável } \\
\text { que ajudou na execução da tarefa. }\end{array}$ & 2,71 & 0,69 & 2,27 & 0,78 & 2,105 & .041 \\
\hline \multicolumn{7}{|l|}{ Desempenho } \\
\hline Eficácia de memorização & 1,96 & 0,62 & 2,85 & 0,37 & $-6,185$ & .001 \\
\hline Eficácia de conteúdo & 2,00 & 0,78 & 2,96 & 0,20 & $-6,084$ & .001 \\
\hline
\end{tabular}

Nota: $\mathrm{gl}=48$ 


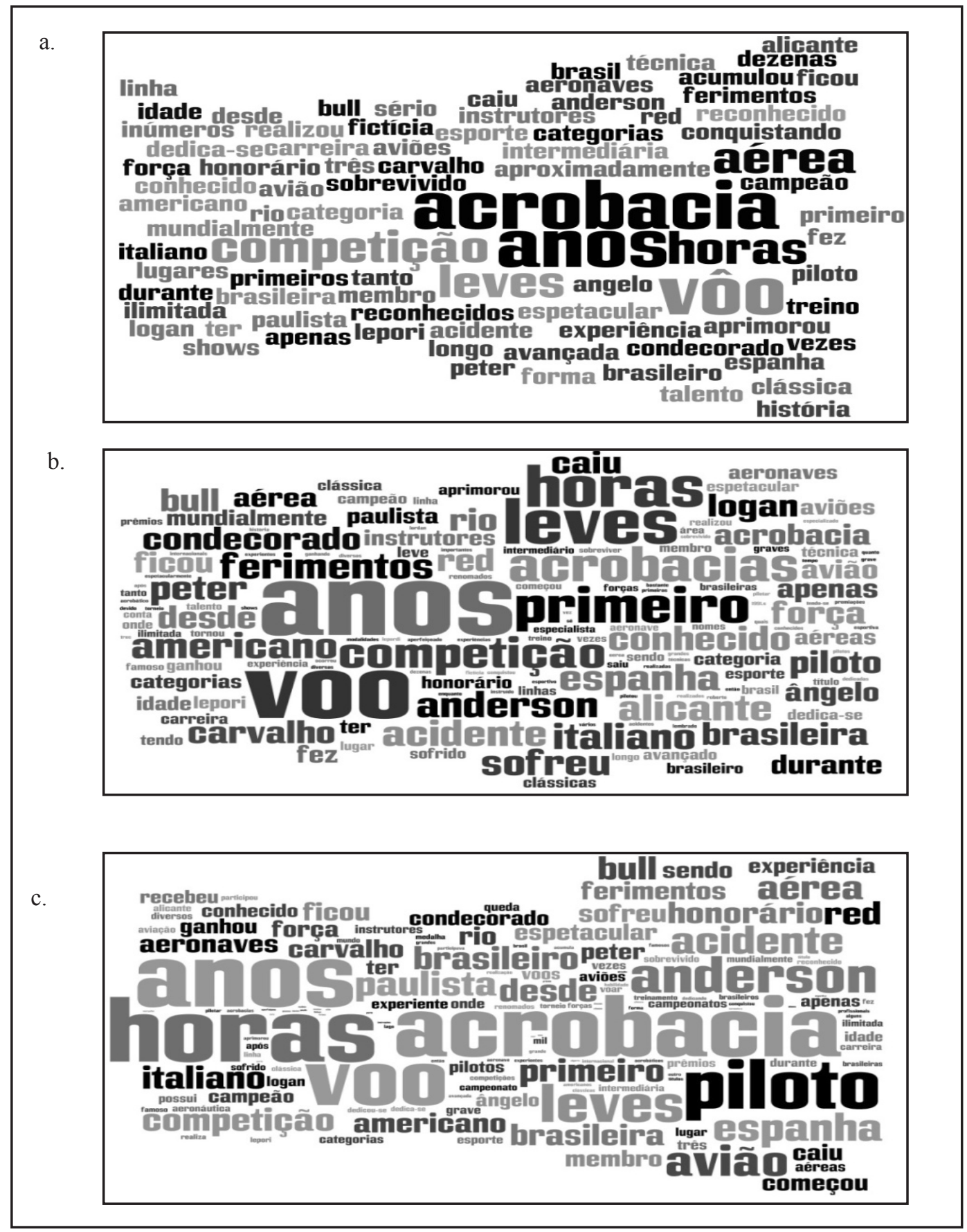

Figura 2. Representações gráficas das frequências das palavras (a) do texto original, (b) no texto da condição grupal e (c) para todos os textos na condição individual.

contribui para ressaltar a importância da tarefa na análise do desempenho de grupo em comparação com o desempenho individual (Puente-Palácios \& Borges-Andrade, 2005).

Crenças sobre o trabalho em equipe

Em relação às crenças sobre trabalho em equipe, a análise de variância, calculada pelo comando Modelo Linear Geral (GLM) do SPSS, item a item, não indicou diferenças significativas, ou seja, independente da condição experimental, a realização da tarefa não resultou em mudança nas crenças sobre o trabalho em equipe. Para a condição grupal, as crenças sobre o trabalho em equipe eram claramente positivas antes da tarefa $(\mathrm{M}=4,95 ; \mathrm{DP}=1,25)$ e se mantiveram assim após a sua realização $(M=4,67$; $\mathrm{DP}=1,09)$. Uma vez mais acredita-se que o tipo de tarefa pode ter contribuído para esse resultado. Uma tarefa mais complexa, provavelmente, poderia levar a desentendimentos entre os membros da equipe sobre a forma de realizar a 
tarefa, colocando em xeque as crenças sobre a eficácia do trabalho em equipe. Em outras palavras, tarefas complexas aumentam as chances de haver conflitos sobre o melhor modo de realizá-las e também sobre a eficácia do grupo (De Dreu \& Weingart, 2003). No caso deste estudo, no entanto, a tarefa foi simples.

Em síntese, as crenças favoráveis ao trabalho em equipe, antes e depois da realização da tarefa, associadas ao bom desempenho da equipe em comparação com o desempenho individual, fortalecem resultados de estudos que afirmam que crenças são preditores de desempenho em equipe (Lent et al., 2006), favorecendo a atuação dos membros do grupo como uma unidade (Watson, Chemers, \& Preiser, 2001).

\section{Percepção de conflitos}

Na condição individual, a percepção de conflitos sobre o trabalho em equipe antes da tarefa $(\mathrm{M}=2,72 ; \mathrm{DP}=0,30)$ praticamente não se alterou após a sua realização $(\mathrm{M}=2,71$; $\mathrm{DP}=0,40)$. Na condição de grupo, por outro lado, a diferença na percepção de conflitos antes $(\mathrm{M}=2,81 ; \mathrm{DP}=0,43)$ e após a realização da tarefa $(\mathrm{M}=2,57 ; \mathrm{DP}=0,47)$ foi estatisticamente significativa $[\mathrm{t}(26)=3,222 ; \mathrm{p}<0,05]$. Esse achado está em sintonia com o resultado encontrado para o afeto positivo "prazer", em que os participantes na condição grupal, após a realização da tarefa, expressaram níveis mais altos deste afeto em relação aos participantes da condição individual. Desse modo, essa vivência mais prazerosa pode se relacionar à ausência ou minimização de conflitos durante a realização da tarefa para a condição grupal.

A redução da percepção de conflito na situação de grupo corrobora os achados de De Dreu e Weingart (2003), que apontam ser o grau de conflito menor para tarefas mais fáceis e rotineiras. Os participantes da condição de grupo, portanto, podem ter considerado simples a tarefa de reprodução de texto, não gerando conflitos sobre o modo de realizá-la.

A percepção de conflitos também pode ser inferida a partir do nível de interação estabelecida para realizar a tarefa. Um dos itens de estratégias de solução de problemas media o nível de interação ("Eu acredito que teria mais êxito na tarefa se houvesse interação virtual com outras pessoas"). Na condição individual, essa afirmativa media a expectativa de que a interação ajudaria no desempenho da tarefa. Na condição de grupo, ao contrário, a afirmativa estava relacionada à crença de que a interação mediada por computadores ajudou no desempenho do grupo. $\mathrm{O}$ resultado foi significativo para $\mathrm{o}$ teste $\mathrm{t}[\mathrm{t}(48)=4,983 ; \mathrm{p}<0,001]$ (Tabela 1 ). Em outras palavras, os participantes que realizaram a tarefa individualmente acreditavam que se tivessem interagido virtualmente teriam alcançado um melhor resultado $(\mathrm{M}=3,46 ; \mathrm{DP}=0,78)$. Ao passo que, na condição grupal $(\mathrm{M}=2,35 ; \mathrm{DP}=0,80)$, a percepção de que a interação colaborou para o desempenho do grupo foi menor.

Uma das conclusões de Gamero et al. (2008) sobre a relação entre conflitos em equipes de trabalho e afetos é que tanto o conflito de relacionamento quanto o de tarefa se relacionaram negativamente com o entusiasmo no nível das equipes. No presente estudo, as evidências apontam nessa direção, pois o entusiasmo se relacionou positivamente com a eficácia de memorização na tarefa $(r=0,31 ; \mathrm{p}<0,05)$, o que pode estar relacionado à baixa percepção de conflitos encontrada após a tarefa.

\section{Auto e heteroatribuições de emoções básicas}

Para analisar as atribuições das emoções básicas (alegria, tristeza, raiva, medo e asco), avaliadas somente após a realização de tarefa com base nas imagens das câmeras de vídeo instaladas em cada computador, criou-se uma nova variável a partir da média de emoções básicas positivas e negativas que foram atribuídas pelos participantes a si mesmos e aos outros participantes de sua sessão. Esse procedimento foi feito tanto para a condição individual quanto grupal. $\mathrm{O}$ teste $\mathrm{t}$ não apresentou diferenças significativas entre condições. Mais uma vez, a suposição é de que o tipo de tarefa pode explicar esse resultado. Ou seja, a tarefa pode ter sido considerada simples e lúdica, não sendo capaz de mobilizar emocionalmente os participantes.

Tanto para participantes alocados nas condições individuais quanto nas condições de grupo, as médias mais altas foram para alegria (individual: $\mathrm{M}=3,7 ; \mathrm{DP}=1,5$; grupal: $\mathrm{M}=4,4 ; \mathrm{DP}=1,6$ ) e as mais baixas para nojo (respectivamente, $\mathrm{M}=1,3 ; \mathrm{DP}=0,7$ e $\mathrm{M}=1,2 ; \mathrm{DP}=1,7)$. Esse resultado permite inferir que, após a realização da tarefa, os participantes de modo geral interpretavam as expressões faciais dos colegas que estavam na sessão como sendo de alegria. Essa seria mais uma evidência de que o tipo de tarefa de memorização não parece ter ativado emoções negativas, como raiva, por exemplo, que seriam reveladoras de conflitos, com repercussões na atribuição de emoções dos colegas (Gamero et al., 2008). Para futuramente vir a ser testado o impacto da tarefa nos estados afetivos dos participantes e sua relação com processos atribuicionais seria necessário introduzir uma tarefa mais complexa que pudesse gerar conflitos sobre o melhor modo de realizar a tarefa. A tarefa de memorização e reprodução proposta no experimento não teve um papel importante na ativação de estados afetivos nos participantes.

\section{Conclusões}

O presente estudo teve o objetivo de analisar as diferenças no desempenho de estudantes universitários em uma tarefa de memorização e reprodução de textos com uso de computadores. A suposição é de que isto poderia ajudar na compreensão de equipes virtuais de trabalho que fazem uso de comunicações mediadas. Testou-se a influência da condição de realização da tarefa (individual ou grupal) sobre o desempenho (eficácia de memorização e de apreensão de conteúdo) e uso de estratégias de solução de problemas. Avaliou-se também se a realização da tarefa modificava as crenças na eficácia do trabalho em equipe, na percepção de conflitos e nos estados afetivos dos participantes.

A principal conclusão do estudo foi que, apesar de os participantes na condição individual terem relatado maior uso de estratégias de solução de problemas, foram os participantes na condição grupal que apresentaram melhor desempenho, tanto em eficácia de memorização quanto 
de apreensão de conteúdo. Isso fortalece as evidências de estudos empíricos que concluem ser a equipe mais eficaz na realização de tarefas (Laughlin \& Ellis, 1986, Potter \& Balthazard, 2002). Em especial, as conclusões deste estudo ajudam a compreender que a colaboração na realização de tarefas mediante uso de computadores, comum em equipes virtuais de trabalho, potencializa o desempenho do grupo. Essa conclusão, no entanto, não significa que equipes virtuais adotem sempre as melhores estratégias que os indivíduos e sejam mais exitosas em termos de desempenho (e.g., Alves \& Gondim , 2014). Estudos com outras amostras e tipos de tarefas poderão avaliar melhor o alcance desses resultados e sua aplicação para o caso de equipes virtuais, cuja forma de colaboração está fortemente centrada na comunicação mediada.

Outra conclusão importante é que a experiência de realização da tarefa em grupo não modificou as crenças positivas sobre o trabalho em equipe, nem aumentou a percepção de conflitos desses participantes. A suposição é de que o tipo de tarefa proposta não propiciou a vivência de situações desafiadoras e de forte intensidade emocional, de forma a diminuir crenças favoráveis ao trabalho em equipe e aumentar a percepção de conflitos interpessoais e de tarefa. Com isso, pouco pode ser explorado neste estudo sobre o papel da tarefa do grupo na mudança de crenças de eficácia e no acirramento de conflitos no grupo.

Acredita-se, portanto, que a principal limitação do estudo tenha sido o tipo de tarefa escolhida, pois não parece ter atingido o objetivo inicial de ativar o sistema de crenças e os estados afetivos dos participantes. Sugere-se que, em estudos futuros, sejam apresentadas tarefas com maior grau de complexidade, de modo a promover a emergência de estados afetivos com maior poder de influenciar as crenças e a percepção de conflitos sobre o trabalho em equipe.

Enfim, apesar dos limites metodológicos e do tamanho da amostra, os resultados aqui apresentados sinalizam a favor da efetividade do grupo na realização de tarefas. Apesar dos limites de generalização desses achados para equipes virtuais, tendo em vista que a amostra foi de estudantes alocados em condição de grupo, e não de equipes de trabalho virtual propriamente dito, a colaboração na realização de tarefas mediante uso de novas tecnologias de informação e comunicação sugerem a importância da natureza da tarefa na efetividade da equipe. Sendo assim, recomenda-se a realização de novos estudos que venham a fazer uso de tarefas mais complexas e igualmente ativadoras de estados afetivos, em um esforço de aproximação das demandas de equipes virtuais nas organizações atuais.

\section{Referências}

Ahuja, M. K., \& Carley, K. M. (1999). Network structure in virtual organizations. Organization Science, 10(6), 741-757.

Alper, E., Tjosvold, D., \& Law, K. S. (2000). Conflict management, efficacy, and performance in organizational teams. Personnel Psychology, 53(3), 625-642.
Alves, G. C., \& Gondim, S. M. G. (2014). Solução de problemas e tomada de decisão em equipes virtuais: um estudo de caso. In J. C. Ribeiro, T. Miranda, \& A. T. Soares (Eds.), Práticas interacionais em rede (pp. 11-28). Salvador: EDUFBA.

Anawati, D., \& Craig, A. (2006). Behavioral adaptation within cross-cultural virtual teams. IEEE Transactions on Professional Communication, 49(1), 44-56.

Bandura, A. (2000). Exercise of human agency through collective efficacy. Current Directions in Psychological Science, 9(3), 75-78.

Bartel, C. A., \& Saavedra, R. (2000). The collective construction of work group moods. Administrative Science Quarterly, 45(2), 197-231.

Barsade, S. (2001). The ripple effect: Emotional contagion and its influence on group behavior. Administrative Science Quarterly, 47(4), 644-675.

Bell, B. S., \& Kozlowski, S. W. (2002). A typology of virtual teams. Group \& Organization Management, 27(1), 14-49.

Bell, S. T., \& Marentette, B. J. (2011). Team viability for long-term and ongoing organizational teams. Organizational Psychology Review, 1(4), 275-292.

Csikszentmihalyi, M., \& LeFevre, J. (1989). Optimal experience in work and leisure. Journal of Personality and Social Psychology, 56(5), 815-822.

De Dreu, C. K. W., \& Weingart, L. R. (2003). Task versus relationship conflict, team performance, and team member satisfaction: A meta-analysis. Journal of Applied Psychology, 88(4), 741-749.

Ekman, P. (1992). Are there basic emotions? Psychological Review, 99(3), 550-553.

Gamero, N., González-Romá, V., \& Peiró, J. M. (2008). The influence of intra-team conflict on work teams' affective climate: A longitudinal study. Journal of Occupational and Organizational Psychology, 81(1), 47-69.

Greenberg, J., \& Baron, R. (1995) Behavior in organizations. Understanding and managing the human side of the work. New Jersey: Prentice-Hall.

Gondim, S. M. G., \& Alvaro, J. L. (2010). Naturaleza y cultura en el estudio de las emociones. Revista Española de Sociología (RES), 13(1), 31-47.

Gondim, S. M. G., \& Mutti, C. M. (2011). Affections in learning situations: A study of an entrepreneurship skills development course. Journal of Workplace Learning, 23(3), 195-208.

Gray, E., \& Watson, D. (2001). Emotion, mood, and temperament: Similarities, differences and a synthesis. In R. L. Payne \& C. L. Cooper (Eds.), Emotions at work: Theory, research and applications for management (pp. 21-44). Chilchester: John Wiley \& Sons.

Griffith, T. L., \& Meader, D. (2004). Prelude to virtual groups: Leadership and technology in semi-virtual groups. In D. Pauleen (Ed.), Virtual teams: Projects, protocols and processes (pp. 231-254). Hershey, PA: Idea Group.

Griffith, T. L., \& Neale, M. A. (2001). Information processing in traditional, hybrid, and virtual teams: From nascent knowledge to transactive memory. In B. M. Staw, \& R. I. Suton (Eds.), Research in organizational behavior (Vol. 23, pp. 379- 421). Stanford CT: JAI Press. 
Guzzo, R. A., \& Shea, G. (1992). Group performance and intergroup relations in organizations. In M. D Dunnette, \& L. M. Hougs (Eds.), Handbook of Industrial and Organization Psychology (Vol. 3, pp. 269-313). Palo Alto, CA: Consulting Psychology Press.

Guzzo, R. A., \& M. W. Dickson. (1996). Teams in organizations: Recent research on performance and effectiveness. Annual Review of Psychology, 47, 307-338.

Hackman, J. R. (1987). The design of work teams. In J. Lorsch (Ed.), Handbook of organizational behavior (pp. 315-342). Englewood Cliffs, NJ: Prentice-Hall.

Harrison, D. A., Mohammed, S., McGrath, J. E., Florey, A. T., \& Vanderstoep, S. W. (2003). Time matters in team performance: Effects of member familiarity, entrainment, and task discontinuity on speed and quality. Personnel Psychology, 56(3), 633-669.

Jehn, K. A. (1994). Enhancing effectiveness: An investigation of advantages and disadvantages of valued based intragroup conflict. International Journal of Conflict Management, 5(3), 223-238.

Kayworth, T. R., \& Leidner, D. E. (2002). Leadership effectiveness in global virtual teams. Journal of Management Information Systems, 18(3), 7-40.

Laughlin, P. R., \& Ellis, A. L. (1986). Demonstrability and social combination processes on mathematical intellective tasks. Journal of Experimental Social Psychology, 22(3), 177-189.

Lent, R. W., Schmidt, J., \& Schmidt, L. (2006). Collective efficacy beliefs in student work teams: Relation to self-efficacy, cohesion, and performance. Journal of Vocational Behavior, 68(1), 73-84.

Martins, M. C. F., Guimarães, V. F., \& Oliveira, M. C. (2006). Adaptação e validação fatorial da Escala de Conflitos Intragrupais. Anais do II Congresso Brasileiro de Psicologia Organizacional e do Trabalho-CBPOT. Brasília, DF.

Martins, M. C. F., \& Puente-Palacios, K. (2010). O psicólogo e sua inserção em equipes de trabalho. In A. V. B. Bastos \& S. M. G. Gondim (Eds.), O trabalho do psicólogo no Brasil (pp. 201-222). Porto Alegre: Artmed.

Potter, R. E., \& Balthazard, P. A. (2002). Virtual team interaction styles: Assessment and effects. International Journal of Human Computer Studies, 56(4), 423-443.

Puente-Palacios, K. E. (2002). Depender ou não depender, eis a questão: um estudo multinível do efeito de padrões de interdependência na satisfação dos membros das equipes de trabalho (Unpublished doctoral dissertation). Universidade de Brasília, Brasília.
Puente-Palacios, K. E., \& Borges-Andrade, J. E. (2005). O efeito da interdependência na satisfação de equipes de trabalho: um estudo multinível. Revista de Administração Contemporânea, 9(3), 57-78.

Puente-Palacios, K. E., \& Albuquerque, F. J. B. (2014). Grupos e equipes de trabalho nas organizações. In J. C. Zanelli, J. E., Borges-Andrade, \& A.V. B. Bastos (Eds.), Psicologia,organizações e trabalho no Brasil (2a ed., pp. 385-412). Porto Alegre: Artmed.

Rangolam, D. I., \& Ballard, D. I. (2007). The meanings and meaningfulness of time in virtual work practices. Retrieved from http://wms-soros.mngt.waikato.ac.nz/ OrganizationalCommunication/Pre-conference.htm.

Saavedra, R., Earley, C., \& Van Dyne, L. (1993). Complex interdependence in Task-Performing Groups. Journal of Applied Psychology, 78(1), 61-72.

Sauaia, A. C. A., \& Zerrener, S. A. (2009). Jogos de empresas e economia experimental: um estudo da racionalidade organizacional na tomada de decisões. Revista de Administração Contemporânea, Curitiba, 13(2), 189-209

Sternberg, R. J. (2000). Psicologia cognitiva. Porto Alegre: Artmed.

Van der Vegt, G., Emans, B., \& Van de Vliert, E. (1999). Effects of interdependencies in project teams. The Journal of Social Psychology, 139(2), 202-214.

Van Der Vegt, G., Emans, B., \& Van De Vliert, E. (2000). Team members' affective responses to patterns of intragroup interdependence and job complexity. Journal of Management, 26(4), 633-655.

Wageman, R. (1995). Interdependence and group effectiveness. Administrative Science Quarterly, 40, 145-180.

Watson, C. B., Chemers, M. M., \& Preiser N. (2001). Collective efficacy: A multilevel analysis. Personality and Social Psychology Bulletin, 27(8), 1057-1068.

West, M., Borrill, C., \& Unsworth, K. (1998). Team effectiveness in organizations. International Review of Industrial and Organizational Psychology, 13(2), 1-48.

Wosnitza, M., \& Volet, S. (2005). Origin, direction and impact of emotions in social online learning. Learning and Instruction, 15(5), 449-464.

Recebido em 05.11.2013

Primeira decisão editorial em 10.01.2014

Versão final em 28.02.2014

Aceito em 27.03.2014 